\title{
On the behavior of mud floc size distribution: model calibration and model behavior
}

\author{
Francesca Mietta - Claire Chassagne • \\ Romaric Verney · Johan C. Winterwerp
}

Received: 15 December 2009 / Accepted: 17 August 2010 / Published online: 22 October 2010

(C) The Author(s) 2010. This article is published with open access at Springerlink.com

\begin{abstract}
In this paper, we study a population balance equation (PBE) where flocs are distributed into classes according to their mass. Each class $i$ contains $i$ primary particles with mass $m_{\mathrm{p}}$ and size $L_{\mathrm{p}}$. All differently sized flocs can aggregate, binary breakup into two equally sized flocs is used, and the floc's fractal dimension is $d_{0}=2$, independently of their size. The collision efficiency is kept constant, and the collision frequency derived by Saffman and Turner (J Fluid Mech 1:16-30, 1956) is used. For the breakup rate, the formulation by Winterwerp (J Hydraul Eng Res 36(3):309-326, 1998), which accounts for the porosity of flocs, is used. We show that the mean floc size computed with the
\end{abstract}

Responsible Editor: Susana B. Vinzon

F. Mietta $(\varangle) \cdot$ C. Chassagne · J. C. Winterwerp Environmental Fluid Mechanics, Faculty of Civil

Engineering and Geosciences, Delft University of Technology, P.O. Box 5048, 2600 GA,

Delft, The Netherlands

e-mail: francescamietta@hotmail.com

C. Chassagne

e-mail: c.chassagne@tudelft.nl

R. Verney

Ifremer, Laboratoire Dyneco/Physed, BP 70,

29280 Plouzane, France

e-mail: romaric.verney@ifremer.fr

J. C. Winterwerp

Deltares, Rotterdamseweg 185, Postbus 177,

2600 MH, Delft, The Netherlands
PBE varies with the shear rate as the Kolmogorov microscale, as observed both in laboratory and in situ. Moreover, the equilibrium mean floc size varies linearly with a global parameter $P$ which is proportional to the ratio between the rates of aggregation and breakup. The ratio between the parameters of aggregation and breakup can therefore be estimated analytically from the observed equilibrium floc size. The parameter for aggregation can be calibrated from the temporal evolution of the mean floc size. We calibrate the PBE model using mixing jar flocculation experiments, see Mietta et al. (J Colloid Interface Sci 336(1):134-141, 2009a, Ocean Dyn 59:751-763, 2009b) for details. We show that this model can reproduce the experimental data fairly accurately. The collision efficiency $\alpha$ and the ratio between parameters for aggregation and breakup $\alpha$ and $E$ are shown to decrease linearly with increasing absolute value of the $\zeta$-potential, both for mud and kaolinite suspensions. Suspensions at high $\mathrm{pH}$ and different dissolved salt type and concentration have been used. We show that the temporal evolution of the floc size distribution computed with this PBE is very similar to that computed with the PBE developed by Verney et al. (Cont Shelf Res, 2010) where classes are distributed following a geometrical series and mass conservation is statistically ensured. The same terms for aggregation and breakup are used in the two PBEs. Moreover, we argue, using both PBEs, that bimodal distributions become monomodal in a closed system with homogeneous sediment, even when a variable shear rate is applied.

Keywords Flocculation - Population balance equation - Discretization into classes $\cdot$ Kaolinite Natural mud $\cdot \zeta$-Potential 


\section{Introduction}

An important parameter in sediment transport modeling is the settling velocity. For cohesive sediment, the settling velocity is affected in a complex way by the sediment properties and the environmental conditions through the process of flocculation (Manning 2001; Eisma 1986). Flocculation can be regarded as a competition between aggregation and breakup. The rate at which flocs grow and the size they attain depend on hydrodynamic conditions, residence time, sediment properties, and properties of the suspension.

The large variability of the floc size and therefore settling velocity of cohesive sediments suggests the need to use different size classes in sediment transport modeling. This may be achieved using computationally efficient flocculation models which allow for the estimation of the temporal evolution of the floc size distribution.

Turbulent motion, at the scale of the floc sizes, governs the collisions frequency between the various flocs and therefore aggregation. However, turbulence also induces pressure differences and differential velocities. These may result in shear and normal stresses, which may disrupt the flocs when they exceed floc strength resulting in floc breakup. Both turbulent motion and stresses can be conveniently quantified by the shear rate $G$ at these small scales (Levich 1962). The shear rate $G$ as defined in Tennekes and Lumley (1972) is therefore commonly used in flocculation modeling. Both models introduced in this paper use $G$ to quantify turbulent motion.

Flocs are considered as fractal objects, which fractal dimension may vary with their size. Maggi (2007) observed that the fractal dimension of kaolinite flocs decreases with the floc size. Similar results have been observed by Khelifa and Hill (2006) on mud flocs from different locations. The average fractal dimension observed for mud flocs is $d_{0}=2$ (Winterwerp 1998).

Flocculation is usually modeled by either simple floc growth equations (i.e., the Lagrangian model developed by Winterwerp 1998, 2002) or by population balance equations (PBEs; Maggi et al. 2007; Serra and Casamitijana 1998). In the first case, the temporal evolution of a characteristic floc size is modeled, while the PBEs model the temporal evolution of a floc size distribution(FSD). For the PBE, we distribute the floc population in different classes according to their size, and the FSD is a vector which accounts for the concentration of flocs in each class. A balance equation is then written for each size class. All balance equations are coupled to a mass conservation equation as the total mass in the system needs to be conserved. Although computationally more demanding, the PBE allows for a better understanding of the processes involved, as it describes the temporal evolution of the FSD.

One of the main differences between the PBEs presented in literature is the way flocs are divided into classes and the method used for integration (Nopens 2005). The division into classes, in fact, affects largely the computational time and the amount of data generated. Only if the computational time and the amount of generated data are small, it is possible to implement the PBE into a sediment transport model. Two different approaches are used in PBEs to ensure mass conservation: (1) the size classes are adapted to the aggregation and breakup mechanisms to ensure mass conservation and (2) the size classes are independent of aggregation and breakup mechanisms and mass conservation is statistically ensured. A more accurate description of the two types of model is given below:

1. The distribution into classes is adjusted to the mechanisms of aggregation and breakup to ensure that there is a size class for each floc resulting from aggregation or breakup. Some examples are the models by Batterham et al. (1981) and Hounslow et al. (1988) in which only aggregation is considered, and the mass of flocs is distributed into classes according to a geometrical series with ratio 2: The mass of each class is twice the mass of the next smaller class. With this distribution into classes, if only equally sized particles can aggregate, the formed flocs belong to the next larger class and each newly formed floc has exactly the mass of the class to which it belongs. If both aggregation and breakup are considered and differently sized particles can aggregate, the number of classes which needs to be considered becomes for this type of models very large as flocs with different sizes may form.

2. Mass conservation is statistically ensured and size classes are defined independently of the mechanisms of aggregation and breakup. Verney developed a model where classes are distributed according to a geometrical series of the floc diameter and mass conservation is ensured by linearly distributing the formed flocs in two neighbor classes, such that the total mass of new flocs equals the mass of the interacting flocs (Verney et al. 2010). Kumar and Ramkrishna (1996) use pivotal points to ensure mass conservation in the system and Zhang and $\mathrm{Li}$ (2003) use the section approximation method. A detailed overview of the different methods is given in Nopens (2005). 
In this paper, we describe a PBE in which size classes are defined on the basis of the number of primary particles in a floc, assuming that all primary particles are solid spheres (fractal dimension $d_{0}=3$ ) with the same mass and density. We refer to this model as PBE 1 in the remainder of this paper. This model is similar to the PBE presented in Maggi et al. (2007) and Mietta et al. (2007), but the algorithm for the numerical integration and the choice of the time step have been improved. PBE 1 follows the first of the two approaches described above: All differently sized particles can aggregate and different breakup distribution functions can be applied to this model. We will show that PBE 1 requires a large number of classes and therefore long computational times. PBE 1 can therefore hardly be used in sediment transport models. Description and behavior of the model are given in Section 2.

In Section 3, we present a simple and efficient algorithm to calibrate the model together with some examples using the experimental data by Mietta et al. (2009a, b). Studies have already been done to link the $\zeta$-potential to the sedimentation behavior of kaolinite (Wang and Siu 2006), or to its rheological properties (Melton and Rand 1977). From these results, a qualitative description of the flocculation behavior has been obtained (Tombacz and Szekeres 2006). The experimental data used for the calibration have been collected to quantify this relation between the flocculation behavior of a suspension and the related $\zeta$-potential. We also briefly observe the relation between the $\zeta$-potential of the suspension and the parameters of the PBE.

We show in Section 4 how PBE 1 can be used to investigate the effect of a reduction in the number of classes on PBE 2 . We compare PBE 1 with the PBE by Verney et al. (2010), called PBE 2 in the remainder of this paper, in which mass conservation is statistically ensured, and classes are divided following to a geometrical series. The same terms for aggregation and breakup are used in PBE 1 and PBE 2. An analysis of the stability of bimodal distributions, using both PBE 1 and PBE 2, is given in Section 5. Discussion and conclusions are given in Section 6.

\section{The population balance equation (PBE 1)}

\subsection{Theory}

We consider a population of fractal flocs, where each floc consists of $i$ primary particles. Assuming that all primary particles have the same mass $m_{\mathrm{p}}$, we discretize the population into $n_{\mathrm{c}}$ classes where the mass of flocs in class $i$ is $m_{i}=i m_{\mathrm{p}} \forall i \in\left[1, n_{\mathrm{c}}\right] . n_{\mathrm{c}}$ is the total number of classes considered. The assumption of equally sized primary particles is rather accurate for pure clay suspensions, for which the primary particles size is rather homogeneous. As natural mud contains both clays and organic matter, the size distribution of mud primary particles is more dispersed. We show later in this paper how the use of equally sized primary particles allows for an accurate representation of experimental data, also for mud suspensions. For a given fractal dimension $d_{0}$, the size of flocs can be computed for each class as $L_{i}=L_{\mathrm{p}} i^{1 / d_{0}}$, where $L_{\mathrm{p}}$ is the size of the primary particles of mass $m_{\mathrm{p}}$. Note that the number of classes to be considered increases sensibly with the size of the flocs, $L_{i}$, as $i=\left(L_{i} / L_{\mathrm{p}}\right)^{d_{0}}$.

In shear-induced flocculation, the size of flocs can hardly exceed the Kolmogorov microscale (Jarvis et al. 2005). If we assume that the largest floc at a given shear rate, $G$, equals the Kolmogorov microscale, we can compute the minimum shear rate for given $n_{\mathrm{c}}, L_{\mathrm{p}}$, and $d_{0}$. For example, if $n_{\mathrm{c}}=2,000, L_{\mathrm{p}}=5 \mu \mathrm{m}$ and $d_{0}=2$, we can resolve all floc sizes for $G_{\lim }=v / \eta^{2}=v / L_{n_{\mathrm{c}}}^{2}=$ $20 \mathrm{~s}^{-1}$ where $v$ is the kinematic viscosity of water, $\eta$ is the Kolmogorov microscale, and $L_{n_{\mathrm{c}}}$ is the size of flocs in class $n_{\mathrm{c}} . L_{\mathrm{p}}$ is the average mean value of kaolinite primary particles distribution shown for kaolinite in Mietta (2010). For any $G>G_{\text {lim }}$, the largest possible flocs are accounted for. We discuss later how also the mean floc size is affected by $n_{\mathrm{c}}$. It is therefore necessary to ensure that the solution of PBE 1 does not depend on $n_{\mathrm{c}}$.

Considering the discretization into classes described above, PBE 1 reads as:

$$
\begin{aligned}
\frac{\Delta N_{i}}{\Delta t}= & \frac{1}{2} \underbrace{\sum_{j=1}^{i-1} \alpha_{j, i-j} \beta_{j, i-j} N_{j} N_{i-j}}_{\mathrm{GA}}-\underbrace{N_{i} \sum_{j=1}^{n_{\mathrm{c}}-i} \alpha_{i, j} \beta_{i, j} N_{j}}_{\mathrm{LA}}-\underbrace{s_{i} N_{i}}_{\mathrm{LB}} \\
& +\underbrace{\sum_{j=i+1}^{n_{\mathrm{c}}} \gamma_{i, j} s_{j} N_{j}}_{\mathrm{GB}},
\end{aligned}
$$

where $\Delta t$ is a finite time step, $\alpha_{i, j}$ is the collision efficiency, $\beta_{i, j}$ is the collision frequency, $s_{i}$ is the breakup rate, and $\gamma_{i, j}$ is the breakup distribution function. This equation is written for all $i \in\left[1, n_{\mathrm{c}}\right]$. The terms GA and LA indicate, respectively, gain and loss by aggregation while the terms GB and LB indicate gain and loss by breakup, respectively. 
The mass conservation equation reads:

$\sum_{i=1}^{n_{\mathrm{c}}} m_{i} N_{i}=c$

In this paper, we presume that the collision efficiency is independent of the size of the interacting particles: $\alpha_{i, j}=\alpha$. This dimensionless parameter is to be assessed through calibration. The collision frequency as derived by Saffman and Turner (1956) is taken into account:

$\beta_{i, j}=\frac{G}{6}\left(L_{i}+L_{j}\right)^{3}$

where $G$ is the shear rate as defined in Tennekes and Lumley (1972). We consider the breakup rate as developed for fractal objects by Winterwerp $(1998,2002)$ :

$s_{i}=E G^{3 / 2}\left(\frac{L_{i}-L_{\mathrm{p}}}{L_{\mathrm{p}}}\right)^{3-d_{0}} L_{i}$.

where $E\left[\mathrm{~s}^{1 / 2} / \mathrm{m}\right]$ is a parameter for breakup to be assessed through calibration. The breakup distribution function, $\gamma_{i, j}$, indicates the number of flocs entering class $i$ as a result of breakup of flocs from class $j$. In this paper, we consider binary breakup in two equally sized flocs only.

PBE 1 is a non-linear system for which an analytical solution cannot be derived. Numerical integration of the system is therefore necessary. We solve the PBE 1 by direct integration: The FSD at each step depends uniquely on the one at the previous step. The time step $\Delta t$ is self-adjustable to ensure that the number of particles leaving a class at time $t$ does not exceed the number of particles in that class at that time.

The numerical simulation is continued until a dynamic equilibrium has been reached using the following criterion:

$\left|L_{\text {mean }, k}-L_{\text {mean }, k-\Delta k}\right| \leq L_{\text {mean }, k} s_{\mathrm{e}}$,

where $\Delta k$ and $s_{\mathrm{e}}$ are conditions for equilibrium, $L_{\text {mean, } k}$ is the average size of the volume-based distribution at the $k$ time step, and $L_{\text {mean, } k-\Delta k}$ is the average size of the volume-based distribution at the $k-\Delta k$ time step. As variations in the mean floc size may be very small, we consider as a criterium for equilibrium the variation of $L_{\text {mean }}$ over $\Delta k$ time steps. This variation should be smaller than a small percentage of the mean value at the time $k, s_{\mathrm{e}} \succeq_{\text {mean, } k}$. Through trial and error, these parameters have been set at $\Delta k=20$ and $s_{\mathrm{e}}=10^{-6}$.

\subsection{Model behavior}

Here we give a concise summary of the behavior of the model. A more detailed description is given in Mietta (2010). All distributions are plotted as volume-based FSD (i.e., the total volume in each class is accounted for), and the mean floc size is computed as:

$L_{\text {mean }}=\frac{\sum_{i=1}^{n_{\mathrm{c}}} L_{i} V_{i} w_{i}}{\sum_{i=1}^{n_{\mathrm{c}}} V_{i} w_{i}}$,

where $V_{i}=v_{i} N_{i}$ with $v_{i}$ volume of flocs in class $i$ is the total volume in class $i$ and $w_{i}$ is the width of the size bin $i$. We compute here the weighted mean, where the width of floc size bins, $w_{i}$, is taken into account, to compare the model results with the experimental data obtained with the Malvern Mastersizer (Mietta et al. 2009a, b). The Malvern software computes a volumebased floc size distribution where flocs are divided into size classes according to a geometrical series. All flocs observed within the edges of a class are grouped into that class. We account therefore for the weighted mean to compare with experimental data. For the comparison between the two models, we will compute the nonweighted mean of the mass-based FSD.

Dynamic equilibrium is reached when aggregation and breakup are in balance. The equilibrium floc size is therefore proportional to the ratio between the rates of aggregation and of breakup. From Eqs. 1, 3, and 4, we find that the rate of aggregation is proportional to:

$\left(\frac{d N_{i}}{d t}\right)_{\mathrm{agg}} \propto \alpha G L_{i}^{3} N_{i}^{2}$

while the rate of breakup is proportional to:

$$
\left(\frac{d N_{i}}{d t}\right)_{\text {bre }} \propto E G^{3 / 2}\left(\frac{L_{i}-L_{\mathrm{p}}}{L_{\mathrm{p}}}\right)^{3-d_{0}} L_{i} N_{i}
$$

The term $\left(\left(L_{i}-L_{\mathrm{p}}\right) / L_{\mathrm{p}}\right)^{3-d_{0}}$ is independent of all parameters with exception of the floc structure (i.e., fractal dimension), which we assume to be invariant. As $d_{0}$ is constant, $L_{i}$ is proportional to $L_{\mathrm{p}}$ as $L_{i}=L_{\mathrm{p}} i^{1 / d_{0}} \propto L_{\mathrm{p}}$. The number of flocs, $N_{i}$, is proportional to the number of primary particles and can be expressed as $N_{i} \propto$ $c /\left(\rho_{\mathrm{s}} L_{\mathrm{p}}^{3}\right)$. The non-dimensional floc size is therefore proportional to:

$L_{\text {mean }}^{*} \equiv \frac{L_{\text {mean }}}{L_{\mathrm{p}}}-1 \propto \frac{\alpha c}{E G^{1 / 2} \rho_{\mathrm{s}} L_{\mathrm{p}}} \equiv P$, 
where $P$ is a non-dimensional global parameter proportional to the rates of aggregation and breakup. We use in this formulation a non-dimensional floc size $L_{\text {mean }}^{*}$, obtained dividing the mean floc size by the size of the primary particles. The term " -1 " has been added to ensure that $L_{\text {mean }}$ equals $L_{\mathrm{p}}$ if there is no aggregation $(P=0)$. In Eq. 9, we observe that the mean floc size in PBE 1 varies with the parameters and physical conditions as the characteristic size computed with the Lagrangian model by Winterwerp (2002). Similar terms for aggregation and breakup have been used in the two models.

The equilibrium $L_{\text {mean }}^{*}$ is plotted as a function of $P$ in Fig. 1a for PBE 1 with different $n_{\mathrm{c}}$. All parameters included in $P$ have been varied independently, and simulations have been done with constant floc structure $d_{0}=2$ and binary breakup. A comparative study of the behavior of the model with constant and variable fractal dimension is shown in Mietta (2010). The mean floc size computed with PBE 1 when $d_{0}=2$ is similar to the one computed with PBE 1 and $d_{0}$ varying with the floc size as in Maggi (2007). The initial growth rate is smaller when the variable fractal dimension is used.

$L_{\text {mean }}^{*}$ varies linearly with $P$ at small $P$. The tangent at the origin of the graph is the same for all $n_{\mathrm{c}}$, and the point $P^{*}$, from which the curve differs from the tangent, increases with $n_{\mathrm{c}}$. For $P>P^{*}, L_{\text {mean }}^{*}$ no longer grows linearly with $P . P^{*} \approx 7$ for $n_{\mathrm{c}}=2,000$, see Fig. 1a.

The equilibrium FSDs for different $P$ are plotted for $n_{\mathrm{c}}=2,000$ in Fig. 1 b. The FSDs at $P>P^{*}=7$ show accumulation of particles in the last class and peaks throughout the distribution. The peaks result from

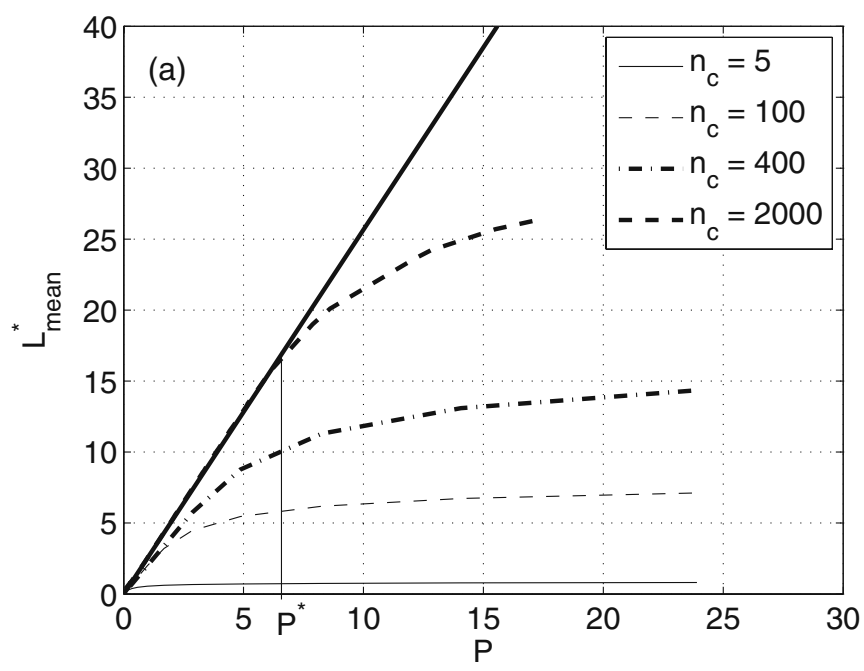

Fig. 1 Equilibrium $L_{\text {mean }}^{*}$ as a function of $P$ for populations with different $n_{\mathrm{c}}$ (a) and FSD at different $P$ for $n_{\mathrm{c}}=2,000(\mathbf{b})$. The thick line in $\mathbf{a}$ is the tangent at small $P$. The FSDs in $\mathbf{b}$ are scaled breakup of flocs in the last class. As binary breakup is considered, the size of each peak is $1 / 2$ the size of the next larger one. This explains why for $P>P^{*}=7$ the relation between $L_{\text {mean* }}$ and $P$ is no longer linear. Then the solution of the PBE 1 is not governed by physics anymore, but it is influenced by the number of classes considered as formed flocs cumulate in the largest class.

For $P<P^{*}$, the relation between $L_{\text {mean }}^{*}$ and $P$ reads as:

$L_{\text {mean }}^{*}=2.567 P$.

This relation holds for all sets of parameters, provided that the fractal dimension, the breakup distribution function, and the aggregation kinetics remain the same. From Eq. 10, the value of $P$ can be computed for each measured equilibrium floc size.

The equilibrium floc size has been observed to vary with the shear rate as the Kolmogorov microscale, both in laboratory experiments (Verney et al. 2010) and in in situ observations (Fettweis et al. 2006; van der Lee et al. 2009). In Fig. 2, $L_{\text {mean }}^{*}$ varies as the Kolmogorov microscale in the range where the solution is not affected by the number of classes considered $n_{\mathrm{c}}$ (high $G$ or low $P$ ). At low shear rate, aggregation dominates over breakup and the equilibrium floc size should increase with decreasing shear rate. If the number of size classes considered is not large enough, flocs cumulate in the largest class and cannot grow further, as shown in Fig. 1. The asymptotic value in Fig. 2 corresponds to the size of the largest class considered.

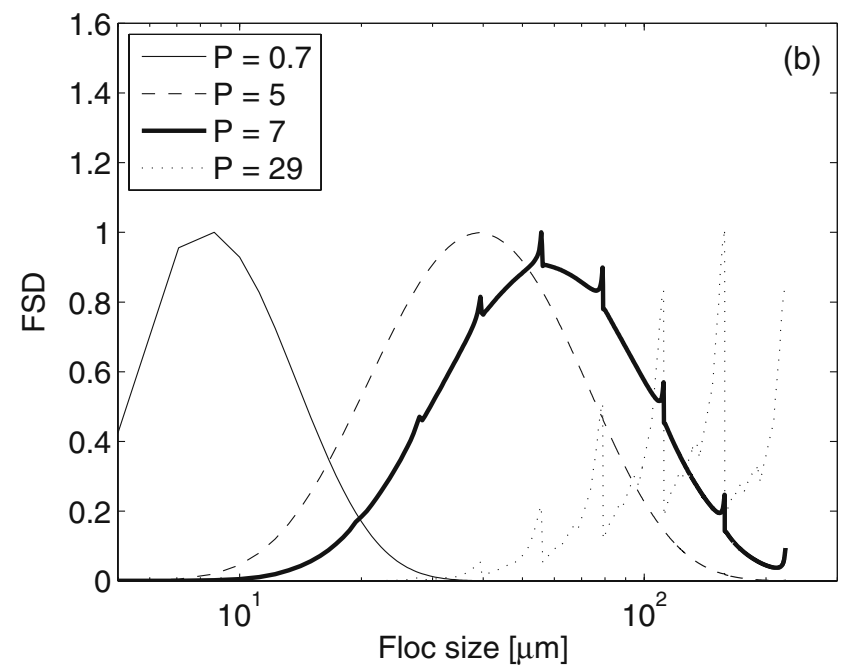

to have the same maximum value so that the plot is easier to read. PBE 1 with $d_{0}=2$ and binary breakup 


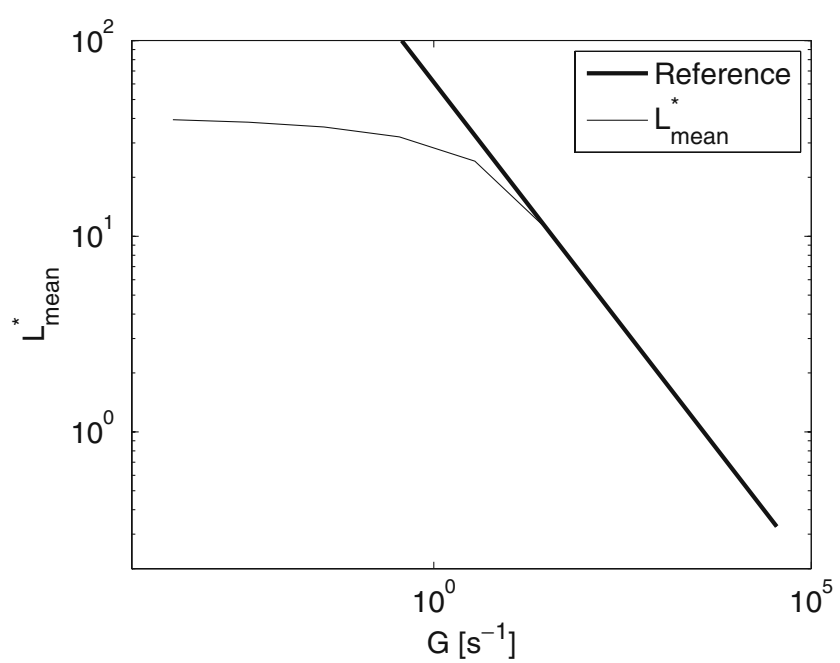

Fig. 2 Equilibrium $L_{\text {mean }}^{*}$ as a function of $G$. The line "reference" scales with $G$ as the Kolmogorov microscale. PBE 1 with $d_{0}=2$ and binary breakup. $L_{\mathrm{p}}=5 \mu \mathrm{m}, \rho_{\mathrm{s}}=2,650 \mathrm{~kg} / \mathrm{m}^{3}$, $c=0.135 \mathrm{~g} / 1, \alpha=0.3$, and $E=0.1 \mathrm{~s}^{1 / 2} / \mathrm{m}$

The breakup and aggregation rates for the PBE 1 have been set such that this ratio scales as $G^{-1 / 2}$. It is shown in Mietta (2010) that this is not sufficient for the computed equilibrium $L_{\text {mean }}^{*}$ to scale with the Kolmogorov microscale. Both the aggregation kinetics and the breakup distribution function influence the way in which $L_{\text {mean }}^{*}$ varies with $G$.

\section{Calibration of the parameters}

\subsection{Approach}

In this section, we calibrate the model using the experimental data presented in Mietta et al. (2009a, b) for kaolinite and mud, respectively. These data have been obtained through mixing jar flocculation experiments using suspensions with different dissolved salts and various concentrations $(\mathrm{pH}>8)$. The $\zeta$-potential of all suspensions has been measured.

To compute the size of flocs from their mass, we use a fractal dimension $d_{0}=2$, which is an average value usually observed in nature (Winterwerp 1998). Mietta (2010) verified that the calibrated parameters vary less than $10 \%$ when the fractal dimension $d_{0}$ is decreased from 2 to 1.7. As the model proved to behave in a similar way for $d_{0}$ larger than $2\left(d_{0}=2.3\right.$; Mietta 2010), we expect a similar behavior when the fractal dimension is increased to values larger than 2 .

Considering that the equilibrium non-dimensional floc size, $L_{\text {mean }}^{*}$, computed with the PBE varies linearly with the parameter $P$ (see Eqs. 6 and 9) and that the temporal evolution depends mainly on the aggregation parameter (Winterwerp 1998), we use the following procedure to assess the model parameters:

- We define $L_{\mathrm{p}}=5 \mu \mathrm{m}$ as the size of the primary particles. This value is close to the mean value of the primary particles size distribution of kaolinite (Mietta 2010). $L_{\mathrm{p}}$ is larger than the smallest floc size observed in experiments; this implies that the behavior of the smallest particles is not modeled by PBE 1. However, only few particles have $L<L_{\mathrm{p}}$.

- From the equilibrium $L_{\text {mean }}$, we compute the value of the parameter $P=L_{\text {mean }}^{*} / 2.567$ as derived from Eq. 10.

- Given $P$, we verify that the number of classes considered, $n_{\mathrm{c}}$, is large enough and that the FSD is independent of $n_{\mathrm{c}}$. We used $n_{\mathrm{c}}=2,000$ for the suspensions with $L_{\text {mean }} \leq 90 \mu \mathrm{m}$ and $n_{\mathrm{c}}=3,000$ for the other suspensions.

- The sediment concentration, $c=0.135 \mathrm{~g} / \mathrm{l}$, the sediment specific weight, $\rho=2,650 \mathrm{~g} / \mathrm{l}$, and the shear rate, $G=35 \mathrm{~s}^{-1}$, are defined by the settings of the experiments. Moreover, we have fixed the size of the primary particles, $L_{\mathrm{p}}$. The ratio between the parameters of aggregation and breakup $\alpha$ and $E$ can therefore be computed from the parameter $P$ as $\alpha / E=P G^{1 / 2} \rho L_{\mathrm{p}} / c$.

- We impose the same initial particle size distribution as observed in the experiments.

- We tune the aggregation parameter $\alpha$ to minimize the error $E_{t}$ between the observed and computed temporal evolution of $L_{\text {mean }}$. $E_{t}$ is defined as:

$E_{t}=\frac{1}{N_{\text {data }}} \sum_{j=1}^{N_{\text {data }}} \frac{\left|L_{\text {mean,data }, t(j)}-L_{\text {mean }, \operatorname{PBE} 1, t(j)}\right|}{\left(L_{\text {mean, data }, t(j)}+L_{\text {mean }, \operatorname{PBE} 1, t(j)}\right) / 2} 100$,

where $N_{\text {data }}$ is the total number of measurements in time during a flocculation experiment, $L_{\text {mean, data, } t(j)}$ is the mean of the measured FSD at the time $t(j)$, and $L_{\text {mean, } \mathrm{PBE} 1, t(j)}$ is the mean of the computed FSD at the same time. We use the Brent algorithm (Press et al. 1989) for the tuning of $\alpha$.

The temporal evolution of $L_{\text {mean }}$ can be divided in two phases (Mietta 2010). In the first phase, aggregation dominates and the growth rate of $L_{\text {mean }}$ is large. In this phase, large flocs are formed. In the second phase, when the size of flocs reaches its maximum and breakup becomes increasingly more important balancing aggregation, the growth rate becomes smaller. This suggests that the parameter 
for aggregation $\alpha$ can be estimated from the temporal evolution of $L_{\text {mean }}$ in the aggregationdominated phase.

To quantify the error in the computed equilibrium FSD, we compute the difference between the computed and measured equilibrium FSDs, $E_{\mathrm{f}}$, as:

$E_{\mathrm{f}}=\frac{1}{n_{\mathrm{c}}} \sum_{k=1}^{n_{\mathrm{c}}} \frac{\left|\mathrm{FSD}_{\text {data,eq }, k}-\mathrm{FSD}_{\mathrm{PBE} 1, \mathrm{eq}, k}\right|}{\left(\mathrm{FSD}_{\text {data, eq }, k}+\mathrm{FSD}_{\mathrm{PBE} 1, \mathrm{eq}, k}\right) / 2} 100$,

where $n_{\mathrm{c}}$ is the number of classes in the FSD, $\mathrm{FSD}_{\text {data,eq, } k}$ is the equilibrium measured FSD in class $k$, and $\mathrm{FSD}_{\mathrm{PBE} 1, \mathrm{eq}, k}$ is the equilibrium computed FSD in class $k$.

\subsection{Results of the calibration}

For all suspensions considered, the calibrated parameters are listed in Table 1 together with the properties of the suspensions and the computed errors.

The collision efficiency is larger for kaolinite suspensions with divalent salt $\left(\mathrm{MgCl}_{2}\right)$ than for suspensions with monovalent salt $(\mathrm{NaCl})$, or sea salt. $\alpha / E$ varies less than the collision efficiency with the properties of the suspension. With exception of the kaolinite suspension with $1,000 \mathrm{mM}$ of added $\mathrm{NaCl}$ at $\mathrm{pH}=9.3, E_{t}$ is below $6 \%$. Few suspensions show high values of $E_{\mathrm{f}}$. An analysis of the effect of the chemical properties of the suspension on flocculation is given in Mietta et al. (2009a, b).

Both the collision efficiency and $\alpha / E$ vary little for the mud suspensions. While the error $E_{t}$ is below $4 \%$, $E_{\mathrm{f}}$ is around $20 \%$ for all mud suspensions. The high $E_{\mathrm{f}}$ values observed depend on the shape of the FSD and will be discussed later.
The temporal evolution of $L_{\text {mean,PBE1 }}$ and $L_{\text {mean,data }}$ is shown in Fig. 3 for a mud suspension with $100 \mathrm{mM}$ of $\mathrm{MgCl}_{2}$ and $\mathrm{pH}=8 . L_{\text {mean,data }}$ and $L_{\text {mean,PBE1 vary }}$ in time in a similar way. The equilibrium floc size is reached after 1 to $2 \mathrm{~h}$ from the beginning of the experiment, and the simulation with PBE 1 well reproduces the temporal evolution of the equilibrium mean size.

Measured and computed FSDs at different times are plotted in Fig. 4 for the suspension considered above. The temporal evolution of the largest classes is the same for both observed and computed FSDs, while the two FSDs are different toward the smaller classes. This is because the size of the primary particles used for $\mathrm{PBE} 1$ is larger than the smallest size measured by the Malvern.

Observed FSDs at equilibrium show a small peak around the size of the primary particles, which is not present in the computed distributions. The shape of the equilibrium FSD obtained through simulation with PBE 1 varies very little with the parameters and is always monomodal. PBE 1 with the settings used for this calibration is therefore not able to generate an equilibrium bimodal FSD as the one observed in the physical experiments. This explains the large errors $E_{\mathrm{f}}$ observed for mud suspensions and for some of the kaolinite suspensions.

We verified in Mietta (2010) that simulations at different shear rates with the parameters calibrated at $G=35 \mathrm{~s}^{-1}$ reproduce the experimental data observed for all shear rates and that the parameters $\alpha$ and $\alpha / E$ are independent from the shear rate $G$.

\subsection{Relation between the physicochemical properties} of the suspension and the model parameters

The aggregation parameter $\alpha$ and the ratio between the aggregation and breakup parameters $\alpha$ and $E$ obtained

Table 1 Parameters and errors for the data used for calibration

\begin{tabular}{|c|c|c|c|c|c|c|c|c|c|}
\hline \multirow[t]{2}{*}{ Sediment } & \multirow[t]{2}{*}{ Salt type } & \multicolumn{2}{|c|}{ Salt concentration } & \multirow[t]{2}{*}{$\mathrm{pH}$} & \multirow{2}{*}{$\begin{array}{l}\zeta \\
\mathrm{mV}\end{array}$} & \multirow{2}{*}{$\begin{array}{l}\alpha \\
-\end{array}$} & \multirow{2}{*}{$\begin{array}{l}\alpha / E \\
\mathrm{~s}^{1 / 2} / \mathrm{m}\end{array}$} & \multirow{2}{*}{$\begin{array}{l}E_{t} \\
\%\end{array}$} & \multirow{2}{*}{$\begin{array}{l}E_{\mathrm{f}} \\
\%\end{array}$} \\
\hline & & $\overline{\mathrm{mM}}$ & $\mathrm{ppt}$ & & & & & & \\
\hline Kaolinite & $\mathrm{MgCl}_{2}$ & 1 & 0.095 & 9.3 & -17.7 & 0.018 & 0.74 & 3.4 & 8.7 \\
\hline Kaolinite & $\mathrm{MgCl}_{2}$ & 100 & 9.5 & 9.3 & -2.5 & 0.099 & 2.41 & 3.8 & 6.8 \\
\hline Kaolinite & $\mathrm{MgCl}_{2}$ & 500 & 47.6 & 9.3 & 8.6 & 0.090 & 3.96 & 6.3 & 13.6 \\
\hline Kaolinite & $\mathrm{NaCl}$ & 100 & 5.8 & 9.3 & -35 & 0.020 & 2.72 & 4.1 & 3.2 \\
\hline Kaolinite & $\mathrm{NaCl}$ & 1,000 & 58 & 9.3 & -5.25 & 0.035 & 6.70 & 18.1 & 16.6 \\
\hline Kaolinite & Sea salt & 79 & 5 & 9.3 & -18.6 & 0.014 & 1.54 & 3.9 & 5.8 \\
\hline Kaolinite & Sea salt & 315 & 20 & 1.8 & -12.3 & 0.010 & 3.50 & 3.3 & 3 \\
\hline Mud & $\mathrm{MgCl}_{2}$ & 100 & 9.5 & 8.0 & -8.8 & 0.141 & 3.92 & 1.2 & 19.7 \\
\hline Mud & $\mathrm{MgCl}_{2}$ & 400 & 38 & 8.0 & 7 & 0.153 & 4.55 & 1.9 & 20.6 \\
\hline Mud & $\mathrm{NaCl}$ & 500 & 29.2 & 8.0 & -13.4 & 0.122 & 3.81 & 1.5 & 18.4 \\
\hline Mud & Sea salt & 315 & 20 & 8.0 & -9.5 & 0.163 & 3.19 & 1.3 & 17.8 \\
\hline Mud & Sea salt & 520 & 33 & 8.0 & -4.8 & 0.176 & 3.17 & 1.5 & 20 \\
\hline
\end{tabular}




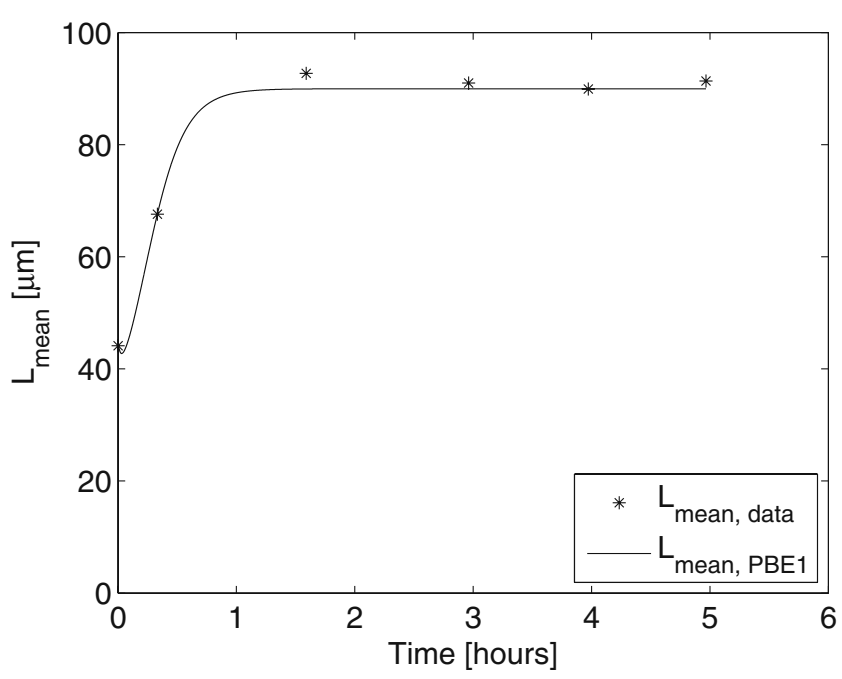

Fig. 3 Temporal evolution of the equilibrium mean size for experimental data $L_{\text {mean,data }}$ and computed values $L_{\text {mean, PBE1 }}$. Sediment concentration $c=0.135 \mathrm{~g} / 1$ and shear rate $G=35 \mathrm{~s}^{-1}$. Mud suspension with $100 \mathrm{mM}$ of $\mathrm{MgCl}_{2}$ and $\mathrm{pH}=8 ; \alpha=0.141$ and $\alpha / E=3.92 \mathrm{~s}^{1 / 2} / \mathrm{m}$

through calibration are plotted as a function of the absolute value of the $\zeta$-potential in Fig. 5a, c for kaolinite and Fig. 5b, $\mathrm{d}$ for mud. We included for $\alpha / E$ more data points than for $\alpha$. These are obtained from a larger set of data where only the equilibrium floc size has been measured, see Mietta et al. (2009a, b) for details.
The collision efficiency of kaolinite suspensions is always smaller than the one of mud, and it increases little when the $\zeta$-potential decreases in magnitude, see Fig. 5a. The increase of the collision efficiency associated to a decrease of the absolute value of the $\zeta$-potential is larger for mud suspensions than for kaolinite suspensions, see Fig. 5b.

The higher collision efficiency for mud suspensions is mainly dependent on the presence of organic matter. Organic matter consists of differently charged polymers which may adhere to particles and enhance flocculation (Winterwerp and van Kesteren 2004). This also explains why the $\zeta$-potential of mud in clear water is smaller in absolute value than the one of kaolinite: The charge of the clay particles may be reduced by the nonor positively charged organic matter adhering to the particles.

The ratio between the parameters $\alpha$ and $E$ computed analytically from the equilibrium floc size increases when the $\zeta$-potential decreases in magnitude, see Fig. 5c, d. The variation of $\alpha / E$ with the $\zeta$-potential is larger for kaolinite than for mud suspensions.

Contrary to what one would expect, considering the complex composition of the mud, both $\alpha$ and $\alpha / E$ correlate better with the $\zeta$-potential for mud suspensions than for kaolinite suspensions. The low correlation for the kaolinite results can be due to the complex
Fig. 4 Temporal evolution of the measured and computed FSD. Sediment concentration $c=0.135 \mathrm{~g} / \mathrm{l}$ and shear rate $G=35 \mathrm{~s}^{-1}$. Mud suspension at $\mathrm{pH}=9.3$ and $100 \mathrm{mM}$ of $\mathrm{MgCl}_{2}, \alpha=0.141$ and $\alpha / E=3.92 \mathrm{~s}^{1 / 2} / \mathrm{m}$. $\mathbf{a} t=20 \mathrm{~min} ; \mathbf{b} t=95 \mathrm{~min}$; c $t=3 \mathrm{~h} ; \mathbf{d} t=5 \mathrm{~h}$
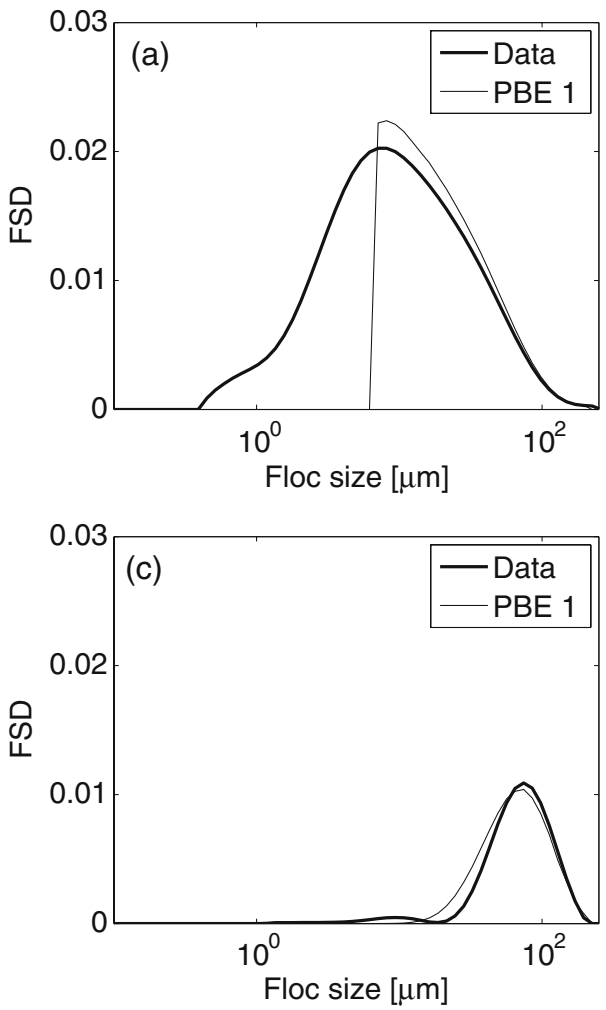
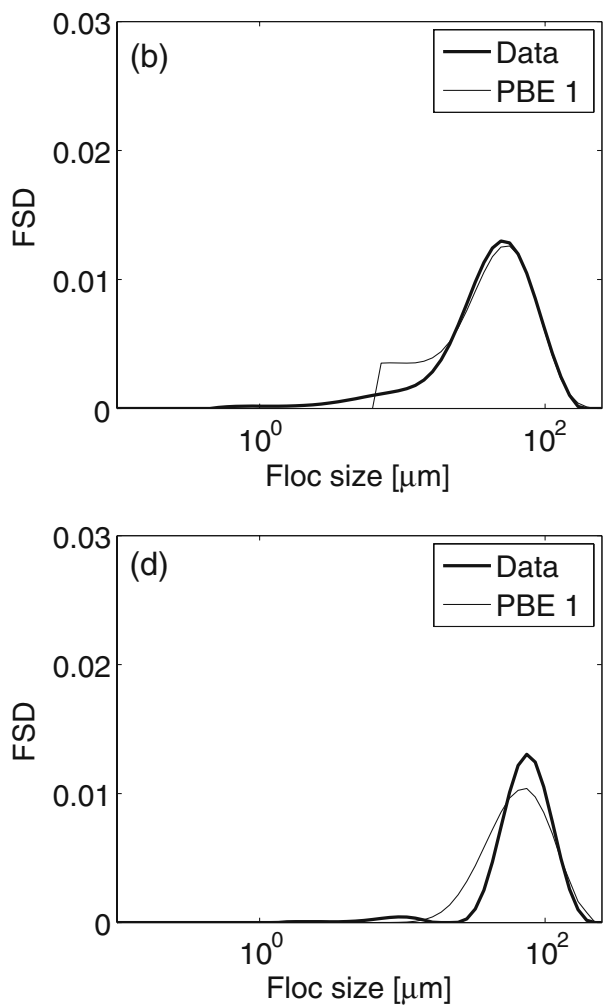

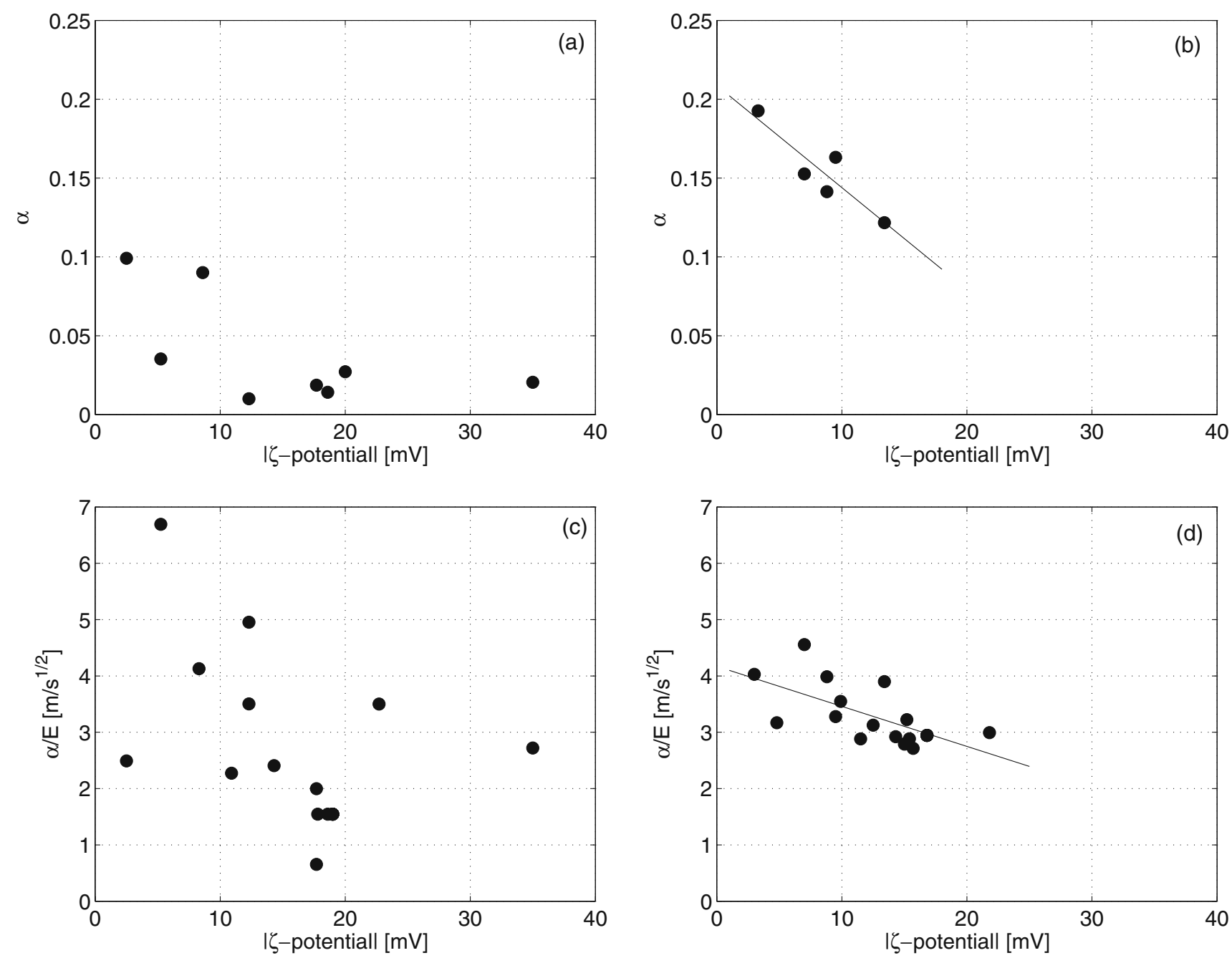

Fig. 5 Collision efficiency $\alpha$ and $\alpha / E$ obtained through calibration of the experimental data. Suspensions at $\mathrm{pH}>8$ and different added salt type and concentration. a $\alpha$, kaolinite suspensions; $\mathbf{b} \alpha$, mud suspensions; $\mathbf{c} \alpha / E$, kaolinite suspensions; d $\alpha / E$, mud suspensions

behavior of this clay, which may chemically interact with dissolved ions (Sposito 1989). Mud is composed of different clays which may interact differently with the dissolved ions. Moreover, organic matter may contribute in smoothing the effect of particles-ions interaction on the flocculation behavior (Mietta et al. 2009b).

\section{Comparison with a PBE with geometrical class division (PBE 2)}

\subsection{Description of PBE 2}

In this section, we describe PBE 2, developed in Verney et al. (2010) and compare it with PBE 1. PBE 2 has been implemented in sediment transport models as it applies a reduced number of classes and is computationally fast.
In PBE 2 are implemented the same terms for aggregation and breakup as in PBE 1. Further, the same parameters and initial distribution have been used for this comparison. The two models differ therefore only for the division into classes and the integration algorithm. Classes for the two models are divided as:

$\begin{cases}L_{i}=L_{\mathrm{p}} i^{1 / d_{0}}, & \text { PBE } 1, \\ L_{i}=L_{\mathrm{p}}^{1+\frac{i-1}{n_{\mathrm{c}}-1}\left(\frac{\log L_{\max }}{\log L_{\mathrm{p}}}-1\right)}, & \text { PBE 2, }\end{cases}$

where $L_{\max }$ is the maximum floc size accounted for in PBE 2. For PBE 1, the maximum floc size which can be resolved depends on the number of classes $n_{\mathrm{c}}$, as the bin's width is kept constant. In PBE 2, on the other hand, the maximum floc size is kept constant and the bin's size depends on the number of classes considered $n_{\mathrm{c}}$. 


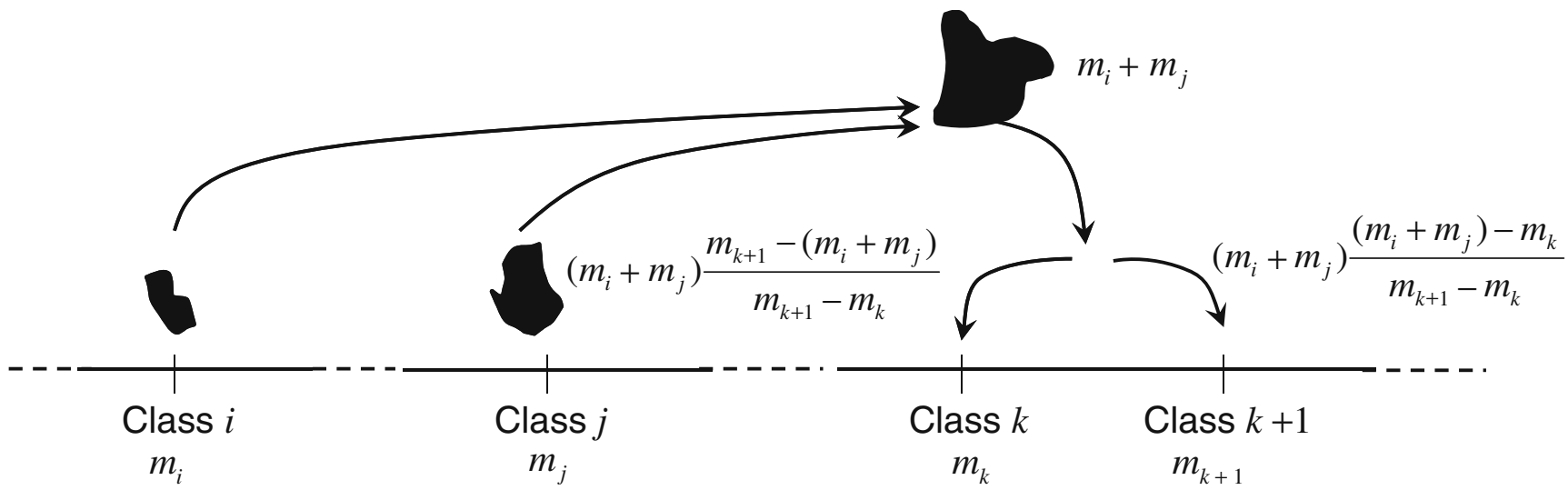

Fig. 6 Management of newly formed flocs in the size class distribution: concept of continuous flocculation (Verney et al. 2010)

Mass conservations are statistically ensured by linearly distributing the flocs resulting from aggregation or breakup in two neighbor classes, see Fig. 6. A detailed description of the behavior of PBE 2 is given in Verney et al. (2010).

For this comparison, we compute for both models the non-weighted mass-based mean value computed as:

$L_{\text {mean }, M}=\frac{\sum_{i=1}^{n_{\mathrm{c}}} L_{i} M_{i}}{\sum_{i=1}^{n_{\mathrm{c}}} M_{i}}$

where $M_{i}$ is the total mass of particles in class $i$. As in this case we do not compare with the experimental data, we do not weight the mean with the bin's size.

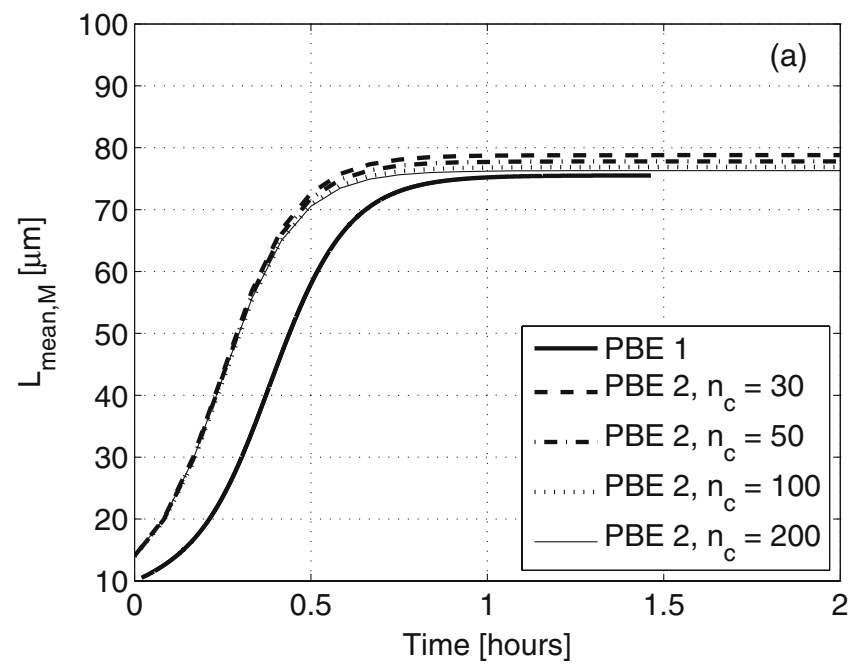

\subsection{Results of the comparison}

The temporal evolution of the mean floc size at $G=$ $35 \mathrm{~s}^{-1}$ is plotted for the two models in Fig. 7. The number of classes and the time step are varied for PBE 2 in Fig. $7 \mathrm{a}, \mathrm{b}$, respectively. $L_{\text {mean }, M}$ computed with PBE 1 is independent of both the time step and $n_{\mathrm{c}}$, if $n_{\mathrm{c}}$ is large enough. Both the equilibrium floc size and the flocculation rate are slightly larger for PBE 2 than for PBE 1.

The equilibrium $L_{\text {mean, } M}$ computed with PBE 2 decreases when the number of classes considered increases. As the size interval between $L_{\mathrm{p}}$ and $L_{\max }$ is divided in $n_{\mathrm{c}}$ classes according to a geometrical series, the width of the bins decreases when $n_{\mathrm{c}}$ increases. When few classes are considered (i.e., $n_{\mathrm{c}}=30$ ), the

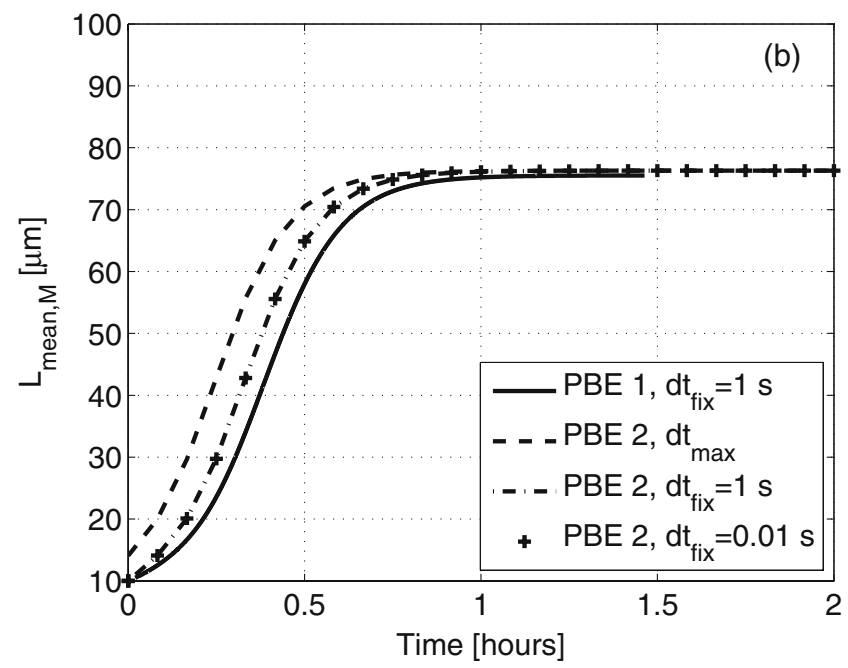

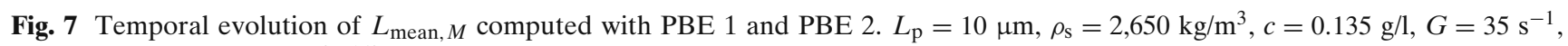
$\alpha=0.359$, and $E=9.510^{-2} \mathrm{~s} / / 2 / \mathrm{m}$. a Variation of $n_{c 2}$ in PBE 2; b variation of the time step in PBE $2, n_{c 1}=4,000$ and $n_{c 2}=200$ 
equilibrium $L_{\text {mean, } M}$ computed with $\mathrm{PBE} 2$ is larger than the one computed with PBE 1 . When the width of the bins approaches the one of PBE $1, L_{\text {mean, } M}$ computed with PBE 2 approaches the one computed with PBE 1, see Fig. 7a. We also note that the temporal evolution is independent of $n_{\mathrm{c}}$.

In Fig. $7 b$, the temporal evolutions of $L_{\text {mean, } M}$ are shown for PBE 1 and for PBE 2 with different time steps. In PBE 1 , an initial time step $d t_{f i x}$ is imposed. This time step may be automatically reduced to ensure that the number of flocs leaving a class because of aggregation and breakup is smaller or equal to the number of flocs in that class for all classes. We verified that the temporal evolution is independent of the initial time step $d t_{f i x}$. The same has been observed for PBE 2 as shown in Fig. 7b. Flocculation is slightly faster for PBE 2 than for PBE 1 . When two particles aggregate in PBE 2, flocs are linearly distributed in two neighbor classes. This implies that large classes are populated rather early in the aggregation process and may explain the larger flocculation rate for PBE 2.

For PBE 2, we show the temporal evolution of $L_{\text {mean, } M}$ also for $d t_{\max }$. This time step is computed at each step as the value for which the number of flocs leaving a class because of aggregation and breakup equals the number of flocs in that class for at least one class and is smaller than the number of flocs in that class for all other classes. Flocculation with $d t_{\max }$ is faster than with $d t_{\text {fix }}$, see Fig. $7 \mathrm{~b}$. Although this is the larger possible time step and allows for reduced

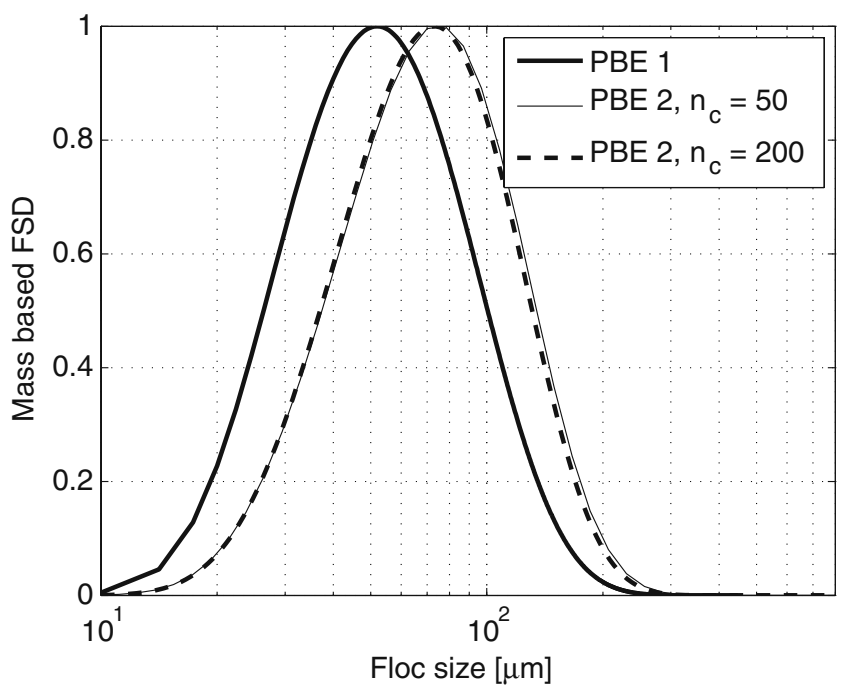

Fig. 8 Equilibrium mass-based FSD computed with PBE 1 and PBE 2. $L_{\mathrm{p}}=10 \mu \mathrm{m}, \rho_{\mathrm{s}}=2,650 \mathrm{~kg} / \mathrm{m}^{3}, G=35 \mathrm{~s}^{-1}, c=0.135 \mathrm{~g} / \mathrm{l}$, $\alpha=0.359$, and $E=9.510^{-2} \mathrm{~s}^{1 / 2} / \mathrm{m}$

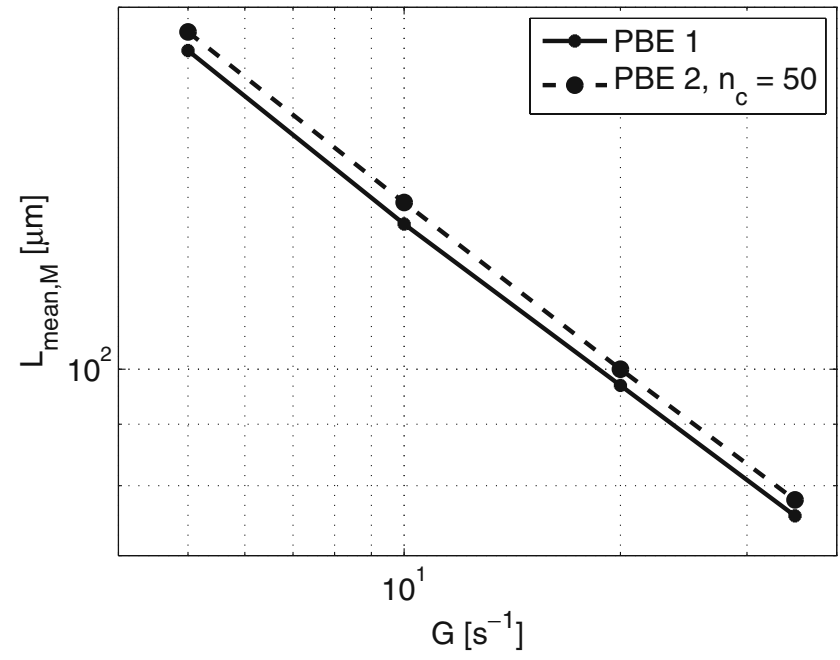

Fig. $9 L_{\text {mean, } M}$ as a function of shear rate computed with PBE 1 and PBE 2. $L_{\mathrm{p}}=10 \mu \mathrm{m}, \rho_{\mathrm{s}}=2,650 \mathrm{~kg} / \mathrm{m}^{3}, c=0.135 \mathrm{~g} / \mathrm{l}$, $\alpha=0.359$, and $E=9.510^{-2} \mathrm{~s}^{1 / 2} / \mathrm{m}$

computational times, it induces a higher flocculation rate than all other time steps.

The FSDs computed with PBE 2 and PBE 1 have the same shape, but the FSD computed with PBE 2 is shifted toward the larger classes, see Fig. 8. This has also been observed for the equilibrium $L_{\text {mean, } M}$, which is larger if computed with PBE 2 than with PBE 1. For PBE 2, the mass concentration in the larger classes is higher when a smaller number of classes is considered. Note that the maximum floc size $L_{\max }$ is smaller than the largest class accounted for in both models. Therefore, the number of classes $n_{\mathrm{c}}$ in PBE 1 does not affect the solution of the model.

The equilibrium $L_{\text {mean, } M}$ varies with the shear rate in the same way for the two models. The values computed with PBE 2 are always larger than for PBE 1 as the number of classes considered in PBE 2 is $n_{\mathrm{c}}=50$, see Fig. 9. Moreover, the ratio between the two $L_{\text {mean }, M}$ is independent of the shear rate $G$ (the distance between the points is constant in a log-log plot).

\section{Stability of a bimodal distribution}

A bimodal floc size distribution is often observed in situ (Manning 2001; Eisma 1986). Different factors may contribute to the formation of a bimodal FSD:

- The variability in time of the shear rate in relation with different flocculation times as a function of the floc size (Eisma 1986; Manning 2001) 
- The composition of the sediment which may include different fractions characterized by different flocculation behavior

- Floc erosion which induces the continuous input of small flocs in the system

- The continuous input of sediment in the water column due to both bed erosion and sediment transport

- The continuous output of sediment due to both sediment transport and deposition

In this section, we discuss the effect of variable shear rate on the stability of a bimodal floc size distribution, using both models introduced in this paper. The effect of the composition of the sediment has been investigated with PBE 1 in Mietta (2010) and the effect of floc erosion, in combination with binary breakup, has been investigated in Verney et al. (2010) with PBE 2. We will come back to this results on the discussion.

To investigate the effect of variable shear rate, $G$ is varied sinusoidally between a minimum and a maximum value. We impose a period of $12 \mathrm{~h}$, similar to a tidal cycle. The initial floc size distribution is bimodal: One of the modes represents the smallest, nonflocculated particles (peak around $15 \mu \mathrm{m}$ ) while the second represents the larger, flocculated particles (peak around $130 \mu \mathrm{m}$ ). The minimum and maximum shear rates used are the ones observed in the Deurgankdok (Western Scheldt, Antwerp, Belgium) by Manning et al. (2007). The parameters for aggregation and breakup are the ones assessed for Western Scheldt mud suspension, see Section 3. The variation in time of the shear rate used in the simulations is plotted in Fig. 10a. The corresponding FSD at different time steps is shown
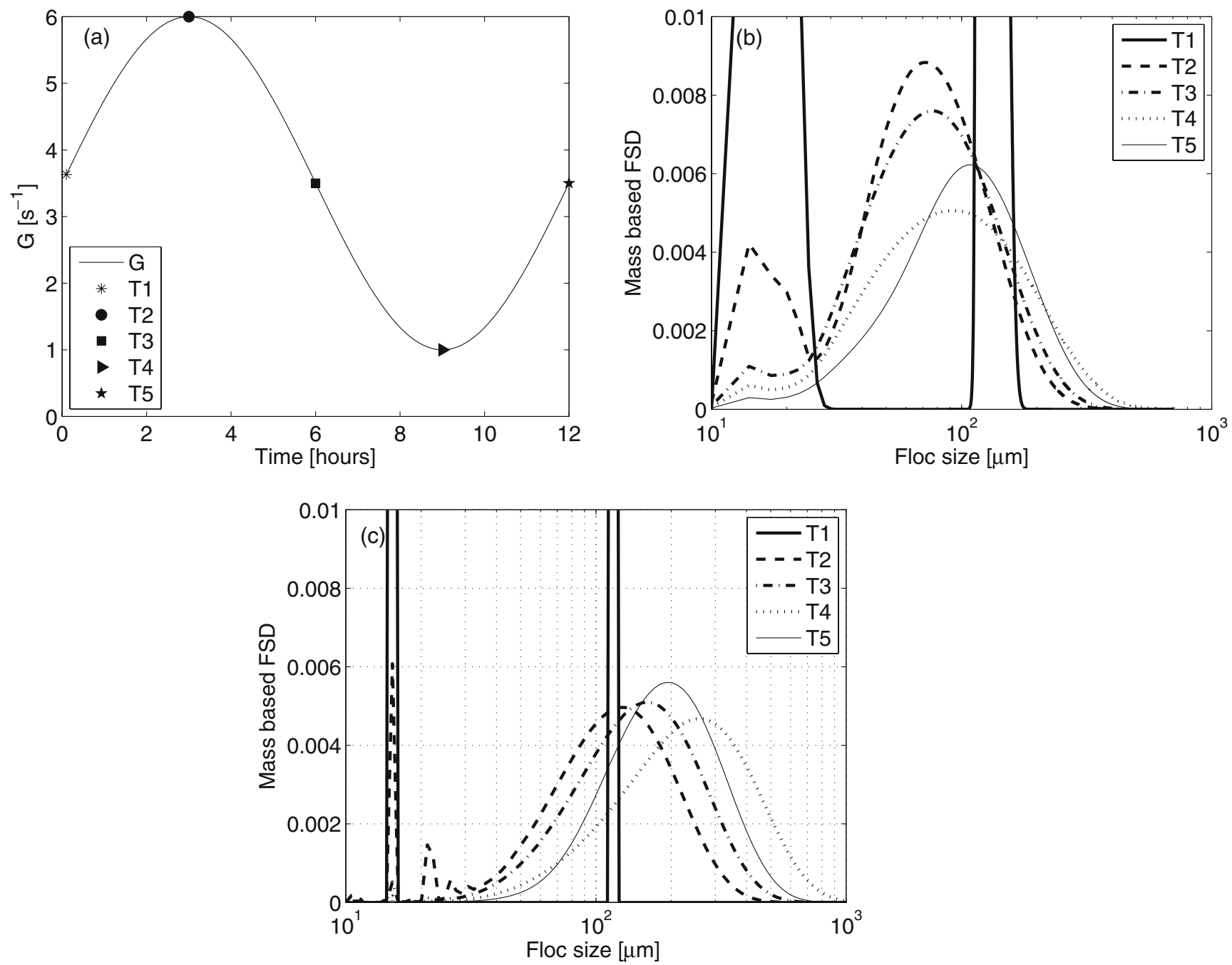

Fig. 10 Temporal evolution of the shear rate $G$ (a) and mass-based FSD at different time steps computed with PBE 1 (b) and PBE 2 (c). $G$ varies sinusoidally with a period of 12 hours, $G_{\min }=1 \mathrm{~s}^{-1}, G_{\max }=6 \mathrm{~s}^{-1}, c=0.135 \mathrm{~g} / \mathrm{l}, \alpha=0.14$, and $E=0.046 \mathrm{~s}^{1 / 2} / \mathrm{m}$ 
for both models in Fig. 10b, c. Five different times have been considered during a tidal cycle to observe the FSD corresponding to different shear values. These times are shown in Fig. 10a.

Both models predict that the peak corresponding to the smaller mode decreases rapidly during the simulation, disappearing within $6 \mathrm{~h}$. The smaller particles aggregate to form larger flocs for all $G$. The maximum floc size and the distribution of the larger flocs vary with the shear rate. From these simple simulations, we may conclude that if all particles have the same flocculation behavior and a closed system is considered, a bimodal distribution cannot be stable. The time to achieve monomodality depends on the parameters for aggregation and breakup, as well as on the sediment concentration and shear rate.

As mentioned above, bimodal distributions observed in situ may depend on other factors. For example, Mietta (2010) accounts for different compositions of the sediment considering that small particles are less likely to aggregate, and they have a smaller collision efficiency. In this case, simulations with PBE 1 show that a bimodal distribution can be conserved over few tidal cycles.

Instead, Verney et al. (2010) tried to explain the stability of bimodal distributions from a combination of binary breakup and erosion. If part of breakup occurs as erosion and part as binary breakup, two peaks are formed in the distribution, the smaller peak is constantly reshaped by the breaking particles, and the initial larger peak oscillates between larger and smaller floc sizes with shear rate.

\section{Conclusions}

The equilibrium mean size computed with the PBE introduced in this paper, PBE 1, increases linearly with the ratio between the rates of aggregation and breakup, $P$. This allows for a one-dimensional calibration of the parameters $\alpha$ and $E$, where the ratio $\alpha / E$ is derived analytically from the equilibrium mean floc size. The collision efficiency $\alpha$, on the other hand, is estimated numerically from the temporal evolution of $L_{\text {mean }}$. We then show that PBE 1 can be successfully used to reproduce the results of mixing jar flocculation experiments. The parameters calibrated for mixing jar flocculation experiments with kaolinite and mud suspensions with different salt type and concentration vary linearly with the $\zeta$-potential. Both the collision efficiency $\alpha$ and the ratio $\alpha / E$ decrease when the $\zeta$-potential increases in absolute value.
The data set used for the calibration has been measured at high shear rate $\left(G=35 \mathrm{~s}^{-1}\right)$. It is shown in Mietta et al. (2009a, b) that these data can be extrapolated to in situ, low shear, conditions using the variation with $G$ of the Kolmogorov microscale. In Mietta (2010), it is also shown that the calibrated parameters are independent of the shear rate $G$. From this, we conclude that this parameters estimated through calibration of data at high shear rate may also refer to in situ conditions.

We show in this paper that two PBEs with the same formulations for aggregation and breakup but different discretization in size classes behave in a very similar way. In PBE 1 , each class $i$ consists of flocs with $i$ primary particles, and mass conservation is straightforward. In the PBE developed by Verney et al. (2010), PBE 2, size classes are distributed following a geometrical series and mass conservation is statistically ensured. Flocculation is slightly faster for PBE 2 than for PBE 1. This may be explained considering that with the mass conservation statistical algorithm in PBE 2, larger classes are occupied earlier in the aggregation process. The equilibrium mean size computed with PBE 2 is very close to that computed with PBE 1 if the number of classes considered in PBE 2 is sufficiently large $\left(n_{\mathrm{c}}=200\right)$ and the width of the size bins is small. When the number of classes considered in PBE 2 decreases, the equilibrium mean value increases. If $n_{\mathrm{c}}=30$ is used for PBE 2, the difference between the equilibrium $L_{\text {mean }}$ computed with the two models is $6 \%$.

From the comparison shown in this paper, we conclude that both PBE 1 and PBE 2 may be used to model flocculation. As mass conservation is straightforward in PBE 1 and no assumption needs to be made to model aggregation and breakup, this model can be used as a reference to test different algorithms for mass conservation used in models such as PBE 2. As mentioned before, the drawbacks of this model are the long computational times and the large amount of data which is generated. PBE 2, on the other hand, is computationally fast and generates a much smaller amount of data. This model can therefore be successfully used for sediment transport modeling. When the number of classes is small, each class can be considered for the estimation of the settling velocity and the accuracy in the calculation of the sediment transport for cohesive sediment is largely increased, although the use of a small number of classes leads to a small error in the computation of $L_{\text {mean }}$. Users should be careful, as an excessive reduction of the number of classes considered may reduce the accuracy of the results, as shown in this paper. 
Simulations done with both models starting from bimodal distributions show that such distributions cannot be stable and that the first mode with smaller flocs tends to disappear in a few hours of simulation. This suggests that bimodal distributions, usually observed in situ, depend on other factors than variable shear rate. We argue that a reason may be the non-homogeneous composition of the sediment, with small, inorganic particles less likely to aggregate than larger organic flocs, or the effect of erosion which has not been treated in this paper. The advection of particles in the domain as well as sediment erosion and settling may also play an important role in the conservation of the bimodal distribution by continuously adding small particles in the first mode.

In this study, we assume that the fractal dimension of flocs, $d_{0}=2$, is independent of their size. It has been observed by different authors, Maggi (2007) and Khelifa and Hill (2006), that the fractal dimension of flocs decreases with increasing floc size. Sonntag and Russel (1987) argue that the strength of flocs and therefore the breakup rate varies with their fractal dimension. Assuming that flocs break always in two equally sized flocs, they relate the floc yield strength to the number of bonds passing through the median plane of the floc. The number of bonds in the median plane of a floc depends on their fractal dimension. These considerations may affect the flocculation rate as well as the equilibrium floc size. Further analysis is necessary on the effect of variable fractal dimension and of fractal dimension's dependent breakup rate on flocculation. Son and Hsu (2009) did a similar study, modeling the temporal evolution of the mean flocs size and observed a smaller flocculation rate with variable fractal dimension and variable floc strength than for constant floc strength.

Acknowledgements This research is supported by the Dutch Technology Foundation STW, applied science division of NWO and the Technology Program of the Ministry of Economic Affairs. The authors are grateful to Deltares for allowing the use of the Malvern instruments.

Open Access This article is distributed under the terms of the Creative Commons Attribution Noncommercial License which permits any noncommercial use, distribution, and reproduction in any medium, provided the original author(s) and source are credited.

\section{References}

Batterham R, Hall J, Barton G (1981) Pelletizing kinetics and simulation of full-scale balling circuits. In: 3rd int. symp. on agglomeration, Nürnberg, W. Germany, pp A136-A150
Eisma D (1986) Flocculation and de-flocculation of suspended matter in estuaries. Neth J Sea Res 20:183-199

Fettweis M, Francken F, Pison V, Van den Eynde D (2006) Suspended particulate matter dynamics and aggregate sizes in a high turbidity area. Mar Geol 235:63-74

Hounslow M, Ryall R, Marshall V (1988) A discretized population balance for nucleation, growth and aggregation. AIChE J 34(11):1821-1832

Jarvis P, Jefferson B, Gregory J, Parsons SA (2005) A review of floc strength and breakage. Water Res 39:3121-3137

Khelifa A, Hill PS (2006) Models for effective density and settling velocity of flocs. J Hydraul Res 44(3):390-401

Kumar S, Ramkrishna D (1996) On the solution of population balance equations by discretisation-I. A fixed pivot technique. Chem Eng Sci 51(8):1311-1332

Levich VG (1962) Physicochemical hydrodynamics. PrenticeHall, Englewood Cliffs

Maggi (2007) Variable fractal dimension: a major control for floc structure and flocculation kinematics of suspended cohesive sediments. J Geophys Res 112:C07012. doi:10.1029/ 2006JC003951

Maggi F, Mietta F, Winterwerp JC (2007) Effect of variable fractal dimension on the floc size distribution of suspended cohesive sediment. J Hydrol 343(1-2):43-55

Manning AJ (2001) A study of the effects of turbulence on the properties of flocculated mud. Ph.D. thesis, Institute of Marine Studies, University of Plumouth, $282 \mathrm{p}$

Manning AJ, Martens C, de Mulder T, Vanlede J, Winterwerp JC, Ganderton P, Graham GW (2007) Mud floc observations in the turbidity maximum zone of the Scheldt estuary during neap tides. J Coast Res 50:832-836

Melton IE, Rand B (1977) Particles interactions in aqueous kaolinite suspensions. II. Comparison of some laboratory and commercial kaolinite samples. J Colloid Interface Sci 60(2):321-330

Mietta F, Maggi F, Winterwerp JC (2007) Effect of the breakup distribution function on the floc size distribution. In: Sediment in ecohydraulics, 2007. Elsevier, Amsterdam

Mietta F, Chassagne C, Winterwerp JC (2009a) Flocculation of kaolinite by turbulent shear as a function of its electrokinetic properties. J Colloid Interface Sci 336(1):134-141

Mietta F, Chassagne C, Manning AJ, Winterwerp JC (2009b) Influence of shear rate, organic matter content, $\mathrm{pH}$ and salinity on mud flocculation. Ocean Dyn 59:751-763. doi:10.1007/ s10236-009-0231-4

Mietta F (2010) Evolution of the floc size distribution of cohesive sediments. Ph.D. thesis, TU Delft, The Netherlands

Nopens I (2005) Modeling the activated sludge flocculation process: a population balance approach. Ph.D. thesis, University of Ghent, Belgium

Press WH, Flammery BP, Teukolsky SA, Vatterling WT (1989) Numerical recipes for Fortran. Cambridge University Press, Cambridge

Saffman PG, Turner JS (1956) On the collision of drops in turbulent clouds. J Fluid Mech 1:16-30

Serra T, Casamitijana X (1998) Modelling the aggregation and breakup of fractal aggregates in a shear flow. Appl Sci Res 59:255-268

Son M, Hsu T-J (2009) The effect of variable yield strength and variable fractal dimension on flocculation of cohesive sediment. Water Res 43:3582-3592

Sonntag RC, Russel WB (1987) Structure and breakup of flocs subjected to fluid stresses. II. Theory. J Colloid Interface Sci 115(2):378-389

Sposito G (1989) The chemistry of soils. Oxford University Press, London 
Tennekes H, Lumley J (1972) A first course into turbulence. MIT, Cambridge

Tombacz E, Szekeres M (2006) Surface charge of kaolinite in aqueous suspension in comparison with montmorillonite. Appl Clay Sci 34:105-124

van der Lee EM, Bowers DG, Kyte E (2009) Remote sensing of temporal and spatial patterns of suspended particle size in the Irish Sea in relation to the Kolmogorov microscale. Cont Shelf Res 29:1213-1225

Verney R, Lafite R, Brun-Cottan JC, Le Hir P (2010) Behaviour of a floc population during a tidal cycle: laboratory experiments and numerical modelling. Cont Shelf Res. doi:10.1016/j.csr.2010.02.005
Wang YH, Siu WK (2006) Structure characteristics and mechanical properties of kaolinite soils. I. Surface charges and structural characterization. Can Geotech J 586-600

Winterwerp JC (1998) A simple model for turbulence induced flocculation of cohesive sediment. J Hydraul Eng Res 36(3):309-326

Winterwerp JC (2002) On the flocculation and settling velocity of estuarine mud. Cont Shelf Res 22:1339-1360

Winterwerp JC, van Kesteren W (2004) Introduction to the physics of cohesive sediments in the marine environment. Development in sedimentology. Elsevier, New York

Zhang J, Li X (2003) Modeling particle-size distribution dynamics in a flocculating system. AIChE J 49(7):1870-1882 